\title{
Scaling of the photon noise in semiconductor cascade lasers
}

\author{
Farhan Rana ${ }^{1}$, Peter Mayer ${ }^{2}$ and Rajeev J Ram ${ }^{2}$ \\ ${ }^{1}$ Department of Electrical and Computer Engineering, Cornell University, Ithaca, NY 14853, \\ USA \\ ${ }^{2}$ Research Laboratory for Electronics, Massachusetts Institute of Technology, Cambridge, \\ MA 02139, USA
}

E-mail: farhan.rana@cornell.edu

Received 17 November 2003, accepted for publication 6 February 2004

Published 27 July 2004

Online at stacks.iop.org/JOptB/6/S771

doi:10.1088/1464-4266/6/8/022

\begin{abstract}
Semiconductor cascade lasers have larger photon noise than conventional semiconductor lasers as a result of positive correlations in photon emission in different gain stages which are connected electrically in series. The photon noise of a cascade laser can be related to the photon noise of a single-stage laser with a scaled external circuit impedance. This scaling relation for the photon noise holds for bipolar as well as unipolar cascade lasers.
\end{abstract}

Keywords: semiconductor lasers, quantum noise, integrated optics

(Some figures in this article are in colour only in the electronic version)

\section{Introduction}

In conventional semiconductor lasers each electron injected into the laser produces at most a single photon. In semiconductor cascade lasers multiple gain stages are connected electrically in series. As a result, each electron injected into the laser is capable of producing multiple photons and the quantum efficiency of a cascade laser can be much larger than $\hbar \omega / e$. The quantum efficiency of a cascade laser with $N$ cascaded stages can therefore be $N$ times the quantum efficiency of a conventional single-stage laser. Short wavelength $(1.55 \mu \mathrm{m})$ interband cascade lasers have potential applications in fibre optic communication networks [1]. Long wavelength $(5-50 \mu \mathrm{m})$ interband and intersubband quantum cascade lasers are the most promising sources of light in the mid- and far infrared regions of the spectrum with potential applications in spectroscopy and imaging [2]. It is therefore important to understand the noise in semiconductor cascade lasers.

Several different types of bipolar and unipolar semiconductor cascade laser have been reported in the literature. Figure 1 shows a bipolar segmented waveguide cascade laser [3] in which a conventional laser waveguide is segmented into smaller sections that are connected electrically in series. Figure 2 shows a bipolar tunnel diode cascade laser $[4,5]$ in which multiple PN junctions are connected electrically in series through reversed biased tunnel diodes. Figure 3 shows a unipolar intersubband quantum cascade laser [2]. In all these devices, different gain stages are connected electrically in series inside the same optical cavity.

In [6] it was shown that the photon emission events in different gain stages of a cascade laser are positively correlated. Immediately after a photon emission event in a gain stage, the carrier density in that gain stage and the potential drop across that gain stage fall below their average values. The circuit responds by pumping extra current to restore the carrier density to its average value. Since the other gain stages are connected electrically in series, the extra current increases the probability of photon emission in all gain stages. The net result is that the emission of a photon in one gain stage increases the probability of photon emission in all the other gain stages. In [7, 8], positive correlations in photon emission in different cascade stages were measured experimentally. The degree of positive correlations in photon emission in different stages depends sensitively on the circuit impedance. A large circuit impedance reduces the ability of the circuit to respond to carrier density fluctuations and, therefore, reduces the positive correlations. In this paper, we analyse the effect of these positive correlations on the photon noise in semiconductor cascade lasers. 

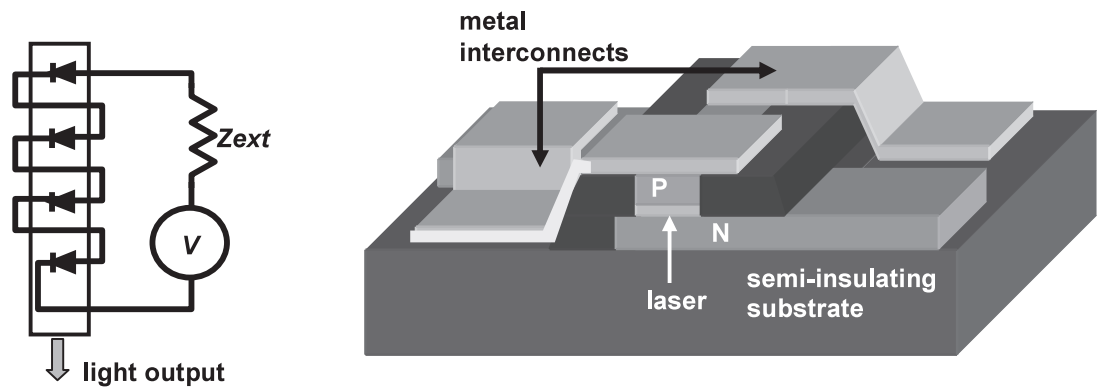

Figure 1. A bipolar segmented waveguide cascade laser [3].
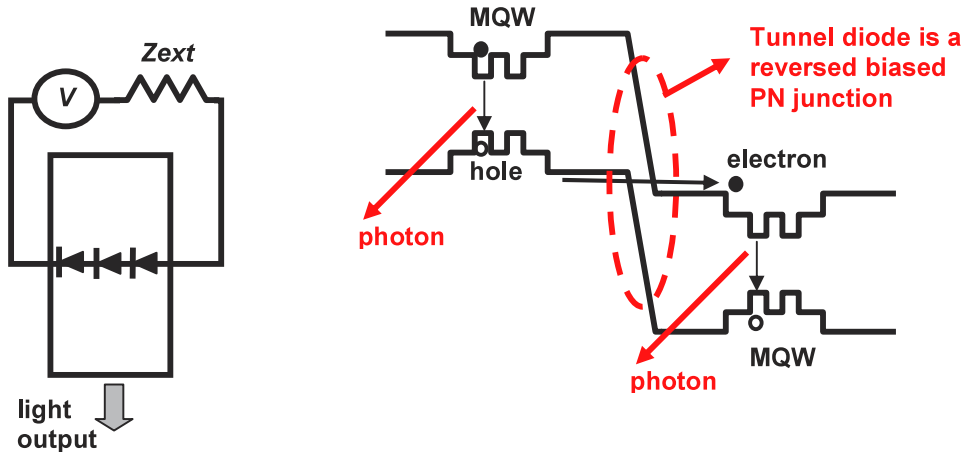

Figure 2. A bipolar tunnel diode cascade laser $[4,5]$.

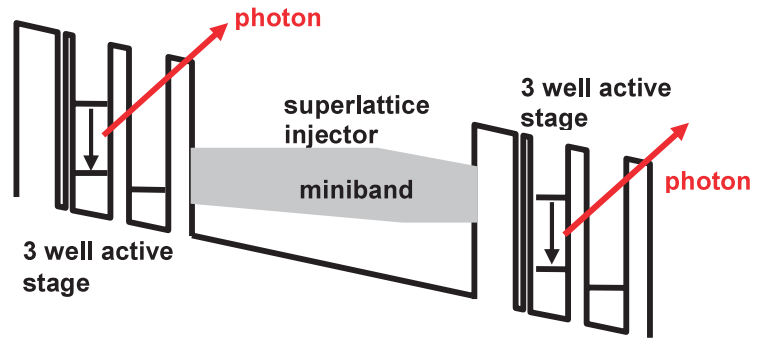

Figure 3. A unipolar quantum cascade laser [2].

\section{Effect of circuit impedance on photon noise in conventional diode lasers}

The photon noise of a conventional single-stage bipolar semiconductor laser also depends on the external circuit impedance. In [9] Yamamoto et al showed that the photon noise of a semiconductor laser can be reduced by increasing the circuit impedance. The effect of external circuit impedance on the photon noise of a semiconductor laser can be explained as follows. Consider a semiconductor laser operating above threshold (figure 4). After each photon emission event the carrier density in the gain stage falls below the average value. The drop in the carrier density reduces the gain and also the probability of photon emission until the carrier density recovers. Therefore, photon noise in a semiconductor laser is regulated by negative feedback from the carrier density. A drop in the carrier density is also accompanied by a drop in the voltage across the active region. The external circuit responds to this drop in the voltage by pumping in extra current that increases the carrier density to its average value. The negative feedback provided by the carrier density is less effective when the circuit impedance is very small since in this case the circuit pumps in large current in response to a voltage change across the gain stage and quickly restores the carrier density in the gain stage to its average value. On the other hand, when the circuit impedance is very large, the circuit is unable to respond to voltage changes across the gain stage, and the carrier density is able to provide negative feedback that helps to suppress the photon noise. The photon noise Fano factor (ratio of the low frequency spectral density of the output photon flux noise to the average photon flux) for a conventional singlestage InP/InGaAsP semiconductor laser is shown in figure 4 as a function of the external circuit impedance. The model for semiconductor laser noise presented in [10] is used in generating figure 4 . The photon noise for small values of the external circuit impedance can be almost an order of magnitude larger than its value for large values of the impedance.

The role of the external circuit impedance in the laser photon noise in a conventional single-stage diode laser can be analysed as follows. Suppose, just after emission of a photon, the voltage across the active region decreases by $\Delta V_{\mathrm{d}}$. The external circuit responds by increasing the current by $\Delta I$, where

$$
\Delta I=\frac{\Delta V_{\mathrm{d}}}{Z_{\mathrm{ext}}+Z_{\mathrm{d}}} .
$$

The increase in current increases the photon emission rate in the laser cavity by $\Delta R$, where

$$
\Delta R=\eta \frac{\Delta I}{e}=\frac{\eta}{e} \frac{\Delta V_{\mathrm{d}}}{Z_{\mathrm{ext}}+Z_{\mathrm{d}}},
$$

$e$ is the electron charge, and $\eta$ is an efficiency factor that relates the increase in the current to the increase in the stimulated emission rate. Equation (2) captures the effect of the circuit impedance on the degree of negative feedback 

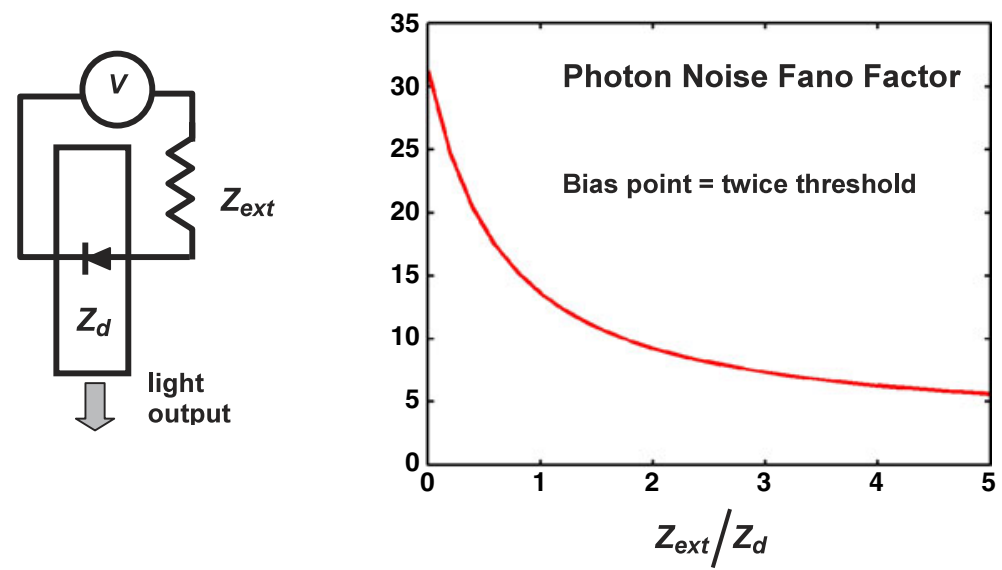

Figure 4. Photon noise versus external impedance for a single-stage semiconductor diode laser.

provided by the carrier density to regulate the photon emission rate. When $Z_{\text {ext }} \ll Z_{\mathrm{d}}$, the circuit is able to provide enough current to quickly restore the carrier density after each photon emission. The carrier density is, therefore, unable to provide negative feedback to regulate the photon emission rate and, consequently, the photon noise in the laser output increases. When $Z_{\text {ext }} \gg Z_{\mathrm{d}}$, the external circuit is unable to provide enough current to quickly restore the carrier density, and the photon noise decreases. It should be noted here that the spectral density $S_{\Delta V_{\mathrm{d}}}(\omega)$ of $\Delta V_{\mathrm{d}}$ is proportional to the total photon emission rate in the laser cavity (or the total laser output power) [9-11]. Above threshold the laser output power is given by the expression [11],

$$
P_{\text {out }}=\eta \frac{\hbar \omega_{0}}{e}\left(I_{\text {bias }}-I_{\text {th }}\right)=\eta \frac{\hbar \omega_{0}}{e} I_{\text {th }}\left(\frac{I_{\text {bias }}}{I_{\text {th }}}-1\right)
$$

where $I_{\text {th }}$ is the threshold current and $I_{\text {bias }}$ is the bias current. The photon noise Fano factor $F\left(I_{\text {bias }} / I_{\text {th }}, Z_{\text {ext }}\right)$ of a semiconductor laser can be expressed as a function of the ratio $I_{\text {bias }} / I_{\text {th }}$ and the circuit impedance $Z_{\text {ext }}[9,10]$. As shown below, the same analysis can be used for semiconductor cascade lasers.

\section{Scaling in semiconductor cascade lasers}

The scaling from a single-stage laser to an $N$-stage cascade laser can be carried out in two ways.

(a) The active region volume of each gain stage is kept constant and, therefore, the total active region volume in the laser cavity is increased by a factor of $N$.

(b) The total active region volume in the laser cavity is kept constant and the active region volume of each gain stage is decreased by a factor of $N$.

Scaling method (a) is used in bipolar tunnel diode cascade and unipolar quantum cascade lasers, and scaling method (b) is used in bipolar segmented waveguide cascade lasers $[2-5,10]$. In method (a), the total impedance of all the gain stages scales as $N Z_{\mathrm{d}}$, where $Z_{\mathrm{d}}$ is the impedance of a single gain stage. In method (b), the volume of the active region of each gain stage is decreased by decreasing its area. Since impedance is inversely proportional to area, the impedance of each gain

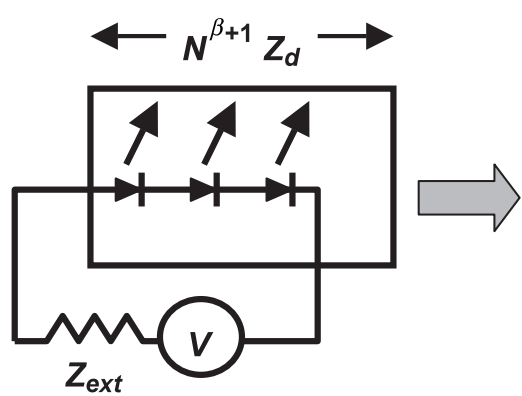

Figure 5. An $N$-stage cascade laser.

stage increases by a factor of $N$, and the total impedance of all the gain stages scales as $N^{2} Z_{\mathrm{d}}$. Therefore, the impedance of a single gain stage scales as $N^{\beta} Z_{\mathrm{d}}$ and the total impedance of all the gain stages scales as $N^{\beta+1} Z_{\mathrm{d}}$ in a cascade laser with $N$ gain stages, where $\beta=0$ for bipolar tunnel diode cascade lasers and unipolar quantum cascade lasers and 1 for bipolar segmented waveguide cascade lasers.

We also make the reasonable assumption that the output power of a cascade laser increases linearly with the increase in the total volume of the laser cavity. This implies that both the output power and the cavity volume scale as $N^{\alpha}$, where $\alpha=1$ for bipolar tunnel diode cascade lasers and 0 for unipolar quantum cascade lasers and bipolar segmented waveguide cascade lasers [3-6]. Since the output power of an $N$-stage cascade laser is given by the expression $[2-6,10]$

$$
P_{\text {out }}=\eta \frac{\hbar \omega_{0}}{e} N\left(I_{\text {bias }}-I_{\text {th }}\right)=\eta \frac{\hbar \omega_{0}}{e}\left(N I_{\text {th }}\right)\left(\frac{I_{\text {bias }}}{I_{\text {th }}}-1\right)
$$

the threshold current $I_{\text {th }}$ and the bias current $I_{\text {bias }}$ must both scale as $N^{\alpha-1}$. The ratio $I_{\text {bias }} / I_{\text {th }}$ does not scale under these assumptions. These are by no means the only possible scaling scenarios but they result in a very simple scaling relation for the photon noise.

\section{Photon noise in semiconductor cascade lasers}

Consider the cascade laser shown in figure 5 with $N$ cascaded gain stages. Suppose that after a photon emission event the voltage across the $j$ th cascade stage decreases by $\Delta V_{\mathrm{d}}^{j}$. The decrease in voltage will increase the circuit current by $\Delta I$, where 


$$
\Delta I=\frac{\Delta V_{\mathrm{d}}^{j}}{Z_{\mathrm{ext}}+N^{\beta+1} Z_{\mathrm{d}}} .
$$

The increase $\Delta R$ in the photon emission rate in the laser cavity as a result of the increase in circuit current is

$$
\Delta R=\eta N \frac{\Delta I}{e}=\frac{\eta}{e} N \frac{\Delta V_{\mathrm{d}}^{j}}{Z_{\mathrm{ext}}+N^{\beta+1} Z_{\mathrm{d}}} .
$$

The factor of $N$ in the numerator in equation (6), in contrast to equation (2), is present because the increase in circuit current increases the photon emission rate in all the cascaded gain stages which are connected electrically in series. The circuit current is therefore responsible for positive correlations in photon emission in different gain stages. Taking into account the independent photon emission events in each gain stage, one can write

$$
\Delta R=\eta N \frac{\Delta I}{e}=\frac{\eta}{e} N \frac{\sum_{j=1}^{N} \Delta V_{\mathrm{d}}^{j}}{Z_{\mathrm{ext}}+N^{\beta+1} Z_{\mathrm{d}}} .
$$

$\Delta V_{\mathrm{d}}^{j}$ contains a scaling factor proportional to $N^{\beta}$ since we assumed that in a cascade laser the area of a single gain stage scales as $N^{\beta}$ and a gain stage with a smaller area will develop a larger voltage drop across it when a single photon is emitted. This scaling can be taken into account explicitly by writing $\Delta V_{\mathrm{d}}^{j}$ as $N^{\beta} \Delta v_{\mathrm{d}}^{j}$. Equation (7) can then be written as

$$
\Delta R=\eta N \frac{\Delta I}{e}=\frac{\eta}{e} \frac{\sum_{j=1}^{N} \Delta v_{\mathrm{d}}^{j}}{\frac{Z_{\mathrm{ext}}}{N^{\beta+1}}+Z_{\mathrm{d}}}=\frac{\eta}{e} \frac{\Delta v_{\mathrm{d}}}{\frac{Z_{\mathrm{ext}}}{N^{\beta+1}}+Z_{\mathrm{d}}}
$$

where $\Delta v_{\mathrm{d}}=\sum_{j=1}^{N} \Delta v_{\mathrm{d}}^{j}$. The spectral density $S_{\Delta v_{\mathrm{d}}}(\omega)$ of $\Delta v_{\mathrm{d}}$ is proportional to the total photon emission rate in the laser cavity. Since the laser output power is assumed to scale as $N^{\alpha}$, the total photon emission rate in the laser cavity will also scale as $N^{\alpha}$. Equation (8) is similar to equation (2) for a single-stage laser and captures the effect of the external circuit impedance on the photon noise. Since the spectral density $S_{\Delta v_{\mathrm{d}}}(\omega)$ and the average photon flux both scale as $N^{\alpha}$, equation (8) implies that the photon noise Fano factor of an $N$-stage cascade laser would be the same as that of a singlestage laser with the external circuit impedance scaled down by $N^{\beta+1}$. Figure 4 shows that for a single-stage laser the photon noise is larger when the external circuit impedance is smaller. Equation (8) shows that the increase in cascade laser noise due to the positive correlations in photon emission in different gain stages can be expressed simply in terms of a scaled external circuit impedance.
More formally, the photon noise Fano factor $F\left(I_{\text {bias }} / I_{\text {th }}\right.$ $\left.Z_{\text {ext }}, N\right)$ of an $N$-stage cascade laser with threshold current $I_{\text {th }}$ bias current $I_{\text {bias }}$, and series impedance $Z_{\text {ext }}$ can be written as

$$
F\left(\frac{I_{\text {bias }}}{I_{\text {th }}}, Z_{\text {ext }}, N\right)=F\left(\frac{I_{\text {bias }}}{I_{\text {th }}}, \frac{Z_{\text {ext }}}{N^{\beta+1}}, 1\right)
$$

where $F\left(I_{\text {bias }} / I_{\text {th }}, Z_{\text {ext }} / N^{\beta+1}, 1\right)$ is the photon noise Fano factor of a single-stage laser with the same value of the ratio $I_{\text {bias }} / I_{\text {th }}$ but with the circuit impedance scaled down to $Z_{\text {ext }} / N^{\beta+1}$

The result in equation (9) can be proved more rigorously for different types of cascade laser even when thermal noise from circuit impedances is included in the model. The details will be published elsewhere. The analysis presented here hides many unnecessary details. Nevertheless, it correctly describes the contribution of the circuit current to the photon noise of lasers. Since the positive correlations in photon emission in different cascade stages come about as a result of the circuit current, the effect of these correlations on the photon noise of cascade lasers is adequately captured in the arguments presented in this paper.

\section{Acknowledgments}

The authors would like to acknowledge the support from DARPA-RFLICS and ONR.

\section{References}

[1] Ram R J, Rana F and Mayer P 2002 Annual Mtg of the IEEE Lasers and Electro-Optics Society (Glasgow, Scotland, Nov. 2002)

[2] Faist J, Tredicucci A, Capasso F and Sirtori C 1998 IEEE J. Quantum Electron. 34336

[3] Getty J T, Buchinsky O, Salvatore R A, Mason B and Coldren L A 1999 Electron. Lett. 351257

[4] Patterson S G, Petrich G S, Ram R J and Kolodzjieski L A 1999 Electron. Lett. 35395

[5] Kim J K, Hall E and Coldren L A 1999 Appl. Phys. Lett. 74 3251

[6] Rana F and Ram R J 2000 Appl. Phys. Lett. 761083

[7] Rana F, Mayer P M and Ram R J 2002 Proc. 18th Semiconductor Laser Conf. (Garmisch, Germany, Sept. 2002) (Piscataway, NJ: IEEE) IEEE Cat. No. 02CH37390

[8] Mayer P, Rana F and Ram R J 2003 Appl. Phys. Lett. 82 689-91

[9] Yamamoto Y and Machida S 1987 Phys. Rev. A 3551145

[10] Rana F and Ram R J 2002 Phys. Rev. B 65125313

[11] Coldren L A and Corzine S W 1995 Diode Lasers and Photonic Integrated Circuits (New York: Wiley) 